\title{
Publisher Correction: RNA-binding proteins contribute to small RNA loading in plant extracellular vesicles
}

Baoye He, Qiang Cai ${ }^{D}$, Lulu Qiao, Chien-Yu Huang, Shumei Wang, Weili Miao, Tommy Ha, Yinsheng Wang and Hailing Jin (D)

Correction to: Nature Plants https://doi.org/10.1038/s41477-021-00863-8, published online 25 February 2021.

The wrong Supplementary Information file was originally published for this Article; it has now been replaced with the correct file.

Published online: 24 March 2021

https://doi.org/10.1038/s41477-021-00901-5

(c) The Author(s), under exclusive licence to Springer Nature Limited 2021 